\title{
Improving Care in the Pediatric Emergency Department With Virtual Reality
}

Jessica DeClercq ${ }^{1}$; Marie Bowen ${ }^{1}$, MEd; Shelia R Cotten ${ }^{1}$, PhD; Aileen Hansen ${ }^{2}$, BS, CCLS, CEIM; Kelly Hebert ${ }^{2}$, BA, CCLS; Jodi Nohel ${ }^{2}$, BRE, CCLS; Kristin Weber ${ }^{2}$, RN; Karl Engler ${ }^{2}$, RN; Kathy Marble ${ }^{2}$, MSN, RN, RNC-NIC

${ }^{1}$ Michigan State University, East Lansing, MI, United States

${ }^{2}$ Sparrow Health System, Lansing, MI, United States

Corresponding Author:

Shelia R Cotten, PhD

Michigan State University

East Lansing, MI,

United States

Email: cotten@msu.edu

\section{Abstract}

Background: Emergency departments (EDs) are often perceived as scary and have been shown to induce anxiety in children. Some hospitals utilize Certified Child Life Specialists (CCLS), hospital staff trained to meet the psychosocial needs of children, to assist when children show signs of anxiety and/or pain during treatment. The CCLS will distract the child, such as with an iPad; however, the child may still watch the IV start, which could result in a failure of the distraction. Digital distraction, distracting a child with technology during stressful procedures, has shown to reduce pain and anxiety better than medications or no distraction at all. Virtual reality (VR) is one form of digital distraction and is increasingly being used in hospitals, as both vision and hearing are blocked by the headset. Previous research shows that the more senses used in the distraction, the less likely the patient will experience pain and anxiety.

Objective: The main goal of this study was to improve care delivery for children in the ED while receiving IV placements. To achieve this, we utilized VR to determine whether it could decrease pain and anxiety for children by acting as a form of digital distraction.

Methods: The intervention included patients between the ages of 5 and 12 who needed an IV in the ED at a public, Michigan hospital. Each participant was randomly assigned to either VR or the standard of care distraction (SD). For those in the VR group, the child played a game while wearing a VR headset. For those in the SD group, the CCLS used standard distraction methods, such as watching a video on an iPad. The guardian then completed a survey to measure the effectiveness and satisfaction of the distraction.

Results: Thirty children participated in the study. Of those who participated, 12 guardians from the VR group and 16 from the SD completed surveys. Seventy-five percent of the VR group and 94 percent of the SD agreed that the distraction reduced the child's anxiety, while 75 percent of the VR group and 88 percent of the SD agreed that the distraction reduced the child's pain during the IV placement. Sixty-seven percent of the VR group versus 94 percent of the SD were more satisfied with health care delivery because of the distraction, and 83 percent of the VR group while 88 percent of the SD were more likely to choose this hospital again because of the distraction.

Conclusions: Although there is potential for the use of VR in health care settings, the use of technology in addition to the CCLS shows great potential to reduce pain and anxiety while improving health care delivery and patient satisfaction. Because most guardians reported positive outcomes with both VR and the SD, the use of technology compared to no distraction should be examined in larger studies to fully understand the effect of digital distraction along with human interaction. With the option of technology in conjunction with caregiver guidance, pediatric patients may perceive less pain and have a better care experience during IV placements in the Emergency Department.

(iproc 2018;4(2):e11796) doi: $10.2196 / 11796$

\section{KEYWORDS}

anxiety; pain; pediatrics; virtual reality 


\section{Multimedia Appendix 1}

Full poster.

[PDF File (Adobe PDF File), 152KB-Multimedia Appendix 1]

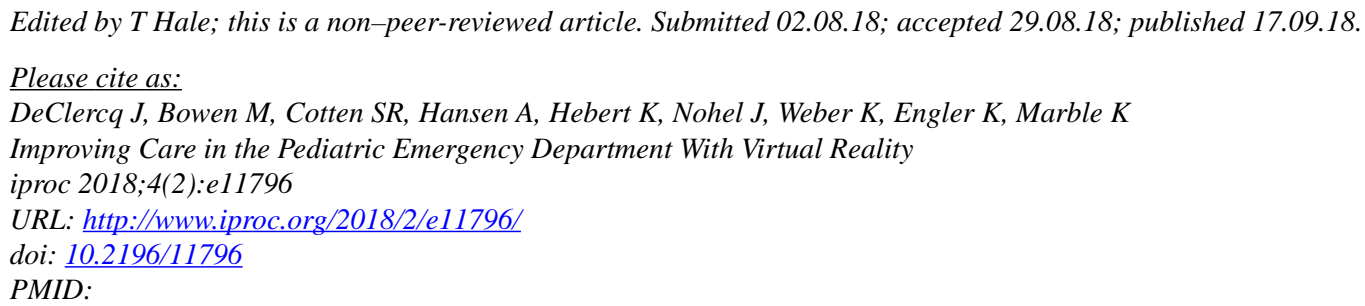

CJessica DeClercq, Marie Bowen, Shelia R Cotten, Aileen Hansen, Kelly Hebert, Jodi Nohel, Kristin Weber, Karl Engler, Kathy Marble. Originally published in Iproceedings (http://www.iproc.org), 17.09.2018. This is an open-access article distributed under the terms of the Creative Commons Attribution License (https://creativecommons.org/licenses/by/4.0/), which permits unrestricted use, distribution, and reproduction in any medium, provided the original work, first published in Iproceedings, is properly cited. The complete bibliographic information, a link to the original publication on http://www.iproc.org/, as well as this copyright and license information must be included. 